\title{
The Assessment of the Knowledge of Health Care Operators on Paliative Care in Vlora Hospital
}

\author{
Mcs. Juljana Xhindoli ${ }^{1}$; RN. Viola Çako ${ }^{2}$; Msc. Mimoza Llavdaniti ${ }^{3}$, MD. Kreshnik Shehu ${ }^{4}$; \\ MD. Julinda Shehu ${ }^{5}$ \\ ${ }^{1}$ Faculty of Public Health, University “I.Qemali” Vlore, Vlore, Albania, \\ ${ }^{2}$ Mp, RN. University “I.Qemali” Vlore; Albania.

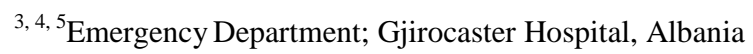

\begin{abstract}
Palliative care is in its infancy in Vlore city and there are no specialized care structures for terminally ill patients in their homes. Their problems are treated in regional hospital, in the pathology ward. Aim: To evaluate the knowledge of health care operators in palliative care in order to determine their informative needs. Methods: In this transversal study we used the "Europall-Palliative-Care-ServicesQuestionnaire"BE-EuroPall. Results: During 2014 in this hospital the staff has provided palliative care for respiratory, oncologic, cardiac and renal diseases. Nurse, medical doctor and specialized oncologist deliver the care. Cooperation with psychologist, physiotherapist and social worker almost do not exist. Two of the most problematic symptoms to manage are respiratory and pain. Most of the physical symptoms of these patients are managed based on subjective evaluation of the professionals, very little on the protocols or experience of colleges, $51 \%$ do not use pain evaluation methods VAS, NRS and WHO Analgesic Scale or they do not even know the scale, $20 \%$ use it always, $26 \%$ sometimes. The staff has started to use the evaluation of the quality care. The personnel need to be informed on physical, bioethics, spiritual aspects of palliative care. Conclusions: Training of the staff on palliative care is an urgent need. The use of evaluation instruments of the patient will raise the quality of the nursing palliative care. It is necessary to develop structures to help the terminally ill patients, in order to treat them with dignity, treat their pain and resolve other problems.
\end{abstract}

Keywords: nursing care, pain evaluation, life quality, knowledge assessment, quality care

\section{Introduction}

Palliative care is in its infancy in Vlora city and there are no specialized care structures for terminally ill patients in their homes. Their problems are treated in regional hospital, in the pathology ward. Management acute and chronic pain remains a persistent problem. These patients are a problem as for his being sick individual but also for their relatives. They require constant care not only for the treatment of pain and other physical problems that are more frequent symptoms in them but also for the care of psychological and spiritual condition of the patient. Palliative treatments aimed at reducing the symptoms of one disease, especially pain as the sufferer for the patient and most annoying for the doctors treating, but also for the family who are interested and care more about them all the time. So in these cases of patients and health conditions, spiritual and psychological (also their family) we must understand that above all palliative care and medication to the patient stand preservation of human dignity which is freedom and independence in actions. These patients have the right to take a good hight quality, at any time. This service should not focus only on treating the physical signs of his patients but complex treatment to have a complete physical welfare mental and [http://www.who.int/cancer/palliative/definition/en/]

\section{Material and Methods}

In this transversal study we used the "Europall-Palliative-CareServices-Questionnaire" [http://www.europall.eu/projectyears-1-3] BE-EuroPall 2010-2013. The study was adapted by European indications and objectives of the study. The inclusion criteria: health staff that is at work across 3 shifts, 4 words in Regional Hospital of Vlora in pathology, emergency, surgery, and reanimation who deal with the treatment of this patient. Questionnaire was anonymous and was explained at the personnel as no "regular" or "wrong" response.

Questionnaire is composed of 3 parts by area: professional and service rale which belongs, to the typology of patients and organizational ways, education and scientific research of operators, developed between 17 questions.

- The first part which contains 4 questions to explore professional role, service that is being belongs, backgrounds/ training for palliative care and cooperation between professionals.

- The second part consists of 7 questions that explore: the type of patients and organizing ways, more assisted pathology, management of problematic symptoms, instruments used to assess the needs of the patient, periodic meetings to discuss cases and measure quality and efficiency concerns.

- The third part consists of 6 questions that explore: taking part in various formations of palliative care, their needs for training in clinical, bioethics, spiritual terms, personal 


\section{International Journal of Science and Research (IJSR) \\ ISSN (Online): 2319-7064 \\ Index Copernicus Value (2013): 6.14 | Impact Factor (2014): 5.611}

interest and on open question based on personal needs for the theme of training required of them.

For questions used dichotomous form of nominal scale (yes/no) or Likert scale (nothing, little, enough, more) with multiple answers and open response questions.

\section{Results}

Replies received from questionnaires filled, in total 39 questionnaires completed by personnel of the regional hospital of Vlora, staff of emergency word (N 14-100\%), pathology department (8 questionnaires), intensive therapy unit (9 questionnaires) and department of surgery (8 questionnaires). 35 questionnaires completed by nurses and 4 questionnaires by doctor. We have no physiotherapist, while in the regional hospital of Vlora are employed only 2 physiotherapists and one psychologist. Any social workers who have to make a multidisciplinary team to achieve quality care in collaboration. Participation in specific training and master of these employees results $24(62 \%)$ of them have followed if a seminar on palliative care, while $9(23 \%)$ of them have attended master (master in pathology, surgery and nursing science), where they received information on palliative cares. But 6(15\%) employees have no taken part in training on palliative care.

Professional images present in your work and to collaborate, and casually they responded as follows: great cooperation is the nurse, oncologist and anesthetist. It noted that images as psychologist, physiotherapist and social worker are to packages to be not saying that do not exist.

Pathologies that are assisted more during 2014(more than one alternative) of respondents to the palliative care are: respiratory pathologies (59\%), oncology (53\%), cardiovascular (23\%), neurological (46\%), renal (43\%) and no-oncology (28\%).

So there is a dominance of respiratory diseases, oncology, cardiovascular and renal that needs palliative care in chronic condition terminals of life.

The management of the main problematic symptoms appears in respiratory difficulty (N-25), pain management (N-24), psychological support (N-24), nausea/vomiting (N-14), constipation/diarrhea $(\mathrm{N}-13)$. So in the management of problematic symptoms belong psychological aspect and the physical, with respiratory difficulty and pain management.

For management of physical symptoms most professionals declares that reference his professional experience, few protocols, little experience of colleagues and no handbook to literature.

According to the respondents instruments used to assess the needs of patients and families, furthermore evaluated physical symptoms and psychological needs but also this in a very low degree and not in all patient (16 from 39 of personnel). Social needs, spiritual, preferences of patient and family to decide, end of life management almost not evaluated.

Use any of the following instruments (if you do, express based on frequency of use)
- The degree of analgesia of WHO-VAS (analog scale of pain), NRS (ruler pain) to assess the level of pain and analgesia rate: Never-20\%, always-20\%, only in some cases-26\%, while 33\% not answered.

For needs assessment of the patient look that over half of the staff 54\% do not use this instrument in pain assessment VAS, NRS and the degree of analgesia of WHO, or they do not know at all as they declared the interviewees, 20\% always use and $26 \%$ many times.

- $\quad$ Evaluation of symptoms; never (N-1), always (N-10), only in some cases (N-2). This assessment is almost unknown to the staff.

- SDS (Symptom Distress Scale) assessing the quality of life shows that: never -3 , always -5 , only in some cases -3 . The evaluation was made by their manner not in scale SDS (Symptom Distress Scale).

- Degree of Braden/Norton to assess the risk of injuries from decubitus, evaluation of symptoms: never $(\mathrm{N}-2)$, Always (N-8), only in some cases $(\mathrm{N}-3)$. The rest of the respondents answered rally that do not know this evaluation.

So how we can see the use of instruments for the evaluation of patients is almost completely unknown, it referred personally by the interviewees or they used a few for the staff that know it.

Did you do periodic meetings to discuss cases and with what frequency?

Noted that personnel treating cases to discuss their problems (82\%-yes and $18 \%$-no), there is no timetable or graphic, but this is at the request of professionals (61\%-yes) so only when they consider it necessary.

Instruments (questionnaire, interview) for measuring the quality/efficiency of the activity in the healthcare system run patients are not used, now they are beginning to be assessed by directorate of hospital and quality impressions to their concerns, it happened only once.

- Taking part in different formations or research in the field of palliative care.

In training and different backgrounds participated interviewed $78 \%$ staff and $22 \%$ they are never trained

a) Needs for training of health personnel in clinical terms are seen to be very necessary especially in:

- Skills in oncology and palliative neurology much-28\%, little $17 \%$, enough $48 \%$, nothing $7 \%$ )

- In the evaluation and management of symptoms (pain, dyspnoea, anorexia, depression, vomiting) much 40\%, enough $33 \%$, little $17 \%$, nothing $10 \%$.

- In the management of severe signs (tumor lesions, fecal incontinence, fistula, skin damage) much 35\%, enough $38 \%$, little $15 \%$, nothing $12 \%$.

- The use of opiates (dose, toxicity, use) much-33\%, enough 33\%, little 19\%, nothing 15\%. 


\section{International Journal of Science and Research (IJSR) \\ ISSN (Online): 2319-7064 \\ Index Copernicus Value (2013): 6.14 | Impact Factor (2014): 5.611}

b) Needs for training of health personnel in terms bioethics seen be necessary especially in: the rights and wishes of the patient, request for euthanasia much-39\%, enough $30 \%$, little $22 \%$, none $9 \%$.

c) While the needs for training of health personnel in spiritual aspect we see more necessary in: the person spiritual condition during treatment much-54\%, enough $30 \%$, little $10 \%$ and nothing $6 \%$.

Open questions based on specific needs proposed by staff with topics of interest are shown on the clinical aspects of pain, therapy, difficult management symptoms, especially appeasing in terminal condition. Personnel needs for training and topics that they would like to receive in-depth knowledge of answered:

- Management and treatment of decubitus, gangrene, wound tumor.

- Management of palliative patient's carcinogen.

- Assessment of pain and its management.

- The meaning of death in our society.

- Psychological management of patients.

- More knowledge on new equipment and in theory.

- More knowledge in palliative care.

- Recent management to life.

- Different protocols on nursing techniques.

\section{Discussions}

Before we begin the discussions it's important to note that the samples that we analyzed is small in the employees of the regional hospital of Vlora, who provide care to chronically ill terminal.

Professional figures that collaborate are nurse, oncologist and anesthetist. Collaboration with professional figures as physiotherapist, psychologist and social worker are too slim, not to say do not exist.

Pathologies that are assisted more during 2014 of respondents to the palliative care are a dominance of respiratory diseases, oncology, cardiovascular and renal that needs palliative care in chronic condition terminals of life.

The management of the main problematic symptoms appears in problematic symptoms belong psychological aspect and the physical, with respiratory difficulty and pain management.

For management of physical symptoms most professionals declares that reference his professional experience, few protocols, little experience of colleagues and no handbook to literature.

According to the respondents instruments used to assess the needs of patients and families, furthermore evaluated physical symptoms and psychological needs but also this in a very low degree and not in all patient. Social needs, spiritual, preferences of patient and family to decide, end of life management almost not evaluated.
Noted that personnel treating cases to discuss their problems, there is no timetable or graphic, but this is at the request of professionals so only when they consider it necessary.

Instruments (questionnaire, interview) for measuring the quality /efficiency of the activity in the healthcare system run patients are not used, now they are beginning to be assessed by directorate of hospital and quality impressions to their concerns, it happened only once.

\section{Conclusions}

Actually in Albania the palliative care is not organized and there are no special structures to care for terminally ill patients at home. In the hospital settings the health care personnel needs to get trained in this particular area. It is necessary to develop multidisciplinary particular structures of the palliative care, with trained staff not only in the physical aspect but also in the spiritual aspect and counseling of the patient's family. The assistance is performed based on the use of instruments for the patient evaluation and WHO recommended protocols for palliative care. It is very important to continually train the staff and improve the palliative care.

\section{Recommendations}

It is necessary to develop the right structures in order to help terminally ill patients, so that they have the proper care, to treat pain and other problems, physical, spiritual and psychosocial ones.

It is necessary to continually train the staff in the field of palliative nursing as a necessity for the staff and the improvement of the nursing care. The use of evaluation instruments would improve the quality of nursing care in palliative patients.

\section{References}

[1] http://www.europall.eu/project-years-1-3

[2] http://www.who.int/cancer/palliative/definition/en/

[3] Henoch I, Axelsson B, Bergman , "The Assessment of Quality of life at the End of Life (AQEL) questionnaire: a brief but comprehensive instrument for use in patients ëith cancer in palliative care", 2010

[4] Lien K, Zeng L, Nguyen, " Comparison of the EORTC QLQ-C15-PAL and the FACIT-Pal for assessment of quality of life in patients ëith advanced cancer", 2011

\section{Author Profile}

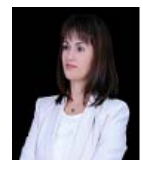

Juljana Xhindoli (Qarri) work as a lecturer at the University of Vlore - Albania, since 2009. Has graduated a General Nurse in University of Vlore in 2001 and M.S. degrees in Vlora University in 2013. She has been a head nurse in the Neonates Unit in Vlore Hospital since 2003-

2009. 